\title{
Multidimensional Multiplicative Combinatorial Properties of Dynamical Syndetic Sets
}

\author{
Jiahao Qiu' • Jianjie Zhao ${ }^{1}$
}

Received: 4 December 2019 / Revised: 2 July 2020 / Accepted: 31 August 2020 / Published online: 7 April 2021

(c) The Author(s) 2021

\begin{abstract}
In this paper, it is shown that for a minimal system $(X, G)$, if $H$ is a normal subgroup of $G$ with finite index $n$, then $X$ can be decomposed into $n$ components of closed sets such that each component is minimal under $H$-action. Meanwhile, we prove that for a residual set of points in a minimal system with finitely many commuting homeomorphisms, the set of return times to any non-empty open set contains arbitrarily long geometric progressions in multidimension, extending a previous result by Glasscock, Koutsogiannis and Richter.
\end{abstract}

Keywords Multidimensional multiplicative large sets · Return times · Decompositions of minimal systems $\cdot$ Minimal systems

Mathematics Subject Classification 37B05 · 05D10

\section{Introduction}

Throughout the paper, a topological dynamical system or just a dynamical system is a pair $(X, G)$, where $X$ is a compact metric space and $G$ acts on it as a group of homeomorphisms. When $G$ is generated by commuting homeomorphisms $T_{1}, \ldots, T_{l}$, we write it as $\left(X, T_{1}, \ldots, T_{l}\right)$. Note that when $l=1$ we recover the classical definition of a dynamical system.

For a classical dynamical system $(X, T)$, the set of return times of a point $x \in X$ to a non-empty open subset $U$ of $X$ is $N(x, U)=\left\{n \in \mathbb{Z}: T^{n} x \in U\right\}$. There

Jiahao Qiu

qiujh@mail.ustc.edu.cn

Jianjie Zhao

zjianjie@mail.ustc.edu.cn

1 Wu Wen-Tsun Key Laboratory of Mathematics, USTC, Chinese Academy of Sciences and School of Mathematics, University of Science and Technology of China, Hefei 230026, Anhui, People's Republic of China 
are many relationships between dynamical properties of the system and the additive combinatorial properties of the sets of return times. For example, if $(X, T)$ is minimal, then every set of return times $N(x, U)$ is syndetic, which means that there exists an integer $h$ such that $N(x, U)$ has non-empty intersection with every interval of $h$ consecutive positive integers. Also we can consider the multiplicative combinatorial properties of the sets of return times. In [9], Glasscock, Koutsogiannis and Richter showed that indeed for a minimal system $(X, T)$, there exists a residual set $X^{\prime}$ such that for any $x \in X^{\prime}$ and any non-empty open subset $U$ of $X$, the set $N(x, U)$ contains arbitrarily long geometric progressions, that is, for any positive integer $h$, there exist positive integers $m, n$ such that $T^{n m} x, \cdots, T^{n m^{h}} x \in U$.

Note that the multiplication in $\mathbb{Z}^{l}$ can also be defined. For $\vec{n}=\left(n_{1}, \ldots, n_{l}\right), \vec{m}=$ $\left(m_{1}, \ldots, m_{l}\right) \in \mathbb{Z}^{l}$, the multiplication $\cdot$ on $\mathbb{Z}^{l}$ can be defined by

$$
\vec{n} \cdot \vec{m}=\left(n_{1}, \ldots, n_{l}\right) \cdot\left(m_{1}, \ldots, m_{l}\right)=\left(n_{1} m_{1}, \ldots, n_{l} m_{l}\right) .
$$

In the current paper, it turns out that the result obtained in [9] can be generalized to $\mathbb{Z}^{l}$. First, we study the minimal system $(X, G)$. Inspired by the idea in [12], it is shown that for any normal subgroup $H$ with finite index in $G, X$ can be decomposed into finite components of closed sets such that each component is minimal under $H$-action (Theorem 3.1). Following this and using the method in [9], for a minimal system $\left(X, T_{1}, \ldots, T_{l}\right)$, we can show that there exists a residual set $X^{\prime}$ such that for any $x \in X^{\prime}$ and any non-empty open subset $U$ of $X$, the set $N(x, U)$ contains arbitrarily long geometric progressions in $\mathbb{N}^{l}$ (Theorem 4.6), which means for any positive integer $h$, there exist $\vec{n}=\left(n_{1}, \ldots, n_{l}\right), \vec{m}=\left(m_{1}, \ldots, m_{l}\right) \in \mathbb{N}^{l}$ such that

$$
\left(T_{1}^{n_{1} m_{1}} \cdots T_{l}^{n_{l} m_{l}}\right) x, \cdots,\left(T_{1}^{n_{1} m_{1}^{h}} \cdots T_{l}^{n_{l} m_{l}^{h}}\right) x \in U
$$

The paper is organized as follows. In Sect. 2, the basic notions used in the paper are introduced. In Sect. 3, a decomposition of a minimal system is given (Theorem 3.1). In Sect. 4, by analysing the dynamics on the orbit closure of the diagonal, we prove that dynamical syndetic sets contain arbitrarily long geometric progressions in multidimension (Theorem 4.6).

\section{Preliminary}

In this section we gather definitions and preliminary results that will be necessary later on. Let $\mathbb{N}\left(\mathbb{Z}, \mathbb{Q}_{+}\right.$, respectively) be the set of all positive integers (integers, positive rational numbers respectively). 


\subsection{Multiplication in $\mathbb{Z}$}

Let $l \in \mathbb{N}$. For $\vec{m}=\left(m_{1}, \ldots, m_{l}\right), \vec{n}=\left(n_{1}, \ldots, n_{l}\right) \in \mathbb{Z}^{l}$, the multiplication $\cdot$ on $\mathbb{Z}^{l}$ is defined by

$$
\vec{m} \cdot \vec{n}=\left(m_{1}, \ldots, m_{l}\right) \cdot\left(n_{1}, \ldots, n_{l}\right)=\left(m_{1} n_{1}, \ldots, m_{l} n_{l}\right) .
$$

Similarly, the multiplication in $\mathbb{N}^{l}$ can also be defined.

We say that $\vec{m}$ is prime to $\vec{n}$, if $\left(m_{i}, n_{i}\right)=1$ for every $i=1, \ldots, l$, which is written as $(\vec{m}, \vec{n})=\overrightarrow{1}$. Put $S_{\vec{n}}=\left\{\vec{m} \in \mathbb{Z}^{l}:(\vec{m}, \vec{n})=\overrightarrow{1}\right\}$.

Let $k \in \mathbb{N}$ and let $\vec{m}_{j}=\left(m_{1}^{(j)}, \ldots, m_{l}^{(j)}\right) \in \mathbb{Z}^{l}, j=1, \ldots, k$. The lowest common multiple of $\vec{m}_{1}, \ldots, \vec{m}_{k}$ is defined by $\vec{M}=\left(M_{1}, \ldots, M_{l}\right) \in \mathbb{N}^{l}$, where every $M_{i}$ is the lowest common multiple of $\left|m_{i}^{(1)}\right|, \ldots,\left|m_{i}^{(k)}\right|$. We write it as $\vec{M}=\operatorname{LCM}\left(\vec{m}_{1}, \ldots, \vec{m}_{k}\right)$.

The set of all residue classes modulo $\vec{m}$ is denoted by $[\vec{m}]$.

Theorem 2.1 (Szemerédi) [11] Let $k \in \mathbb{N}$ and $0<\varepsilon<1$, then there exists $L \in \mathbb{N}$ such that every subset $A$ of $\{1, \ldots, L\}$ with $|A|>\varepsilon L$ contain an arithmetic progression of length $k$.

Proposition 2.2 Let $k, l \in \mathbb{N}, \vec{m} \in \mathbb{N}^{l}$ and $0<\varepsilon<1$, then there exists $L \in \mathbb{N}$ such that for all subsets $A$ of $\left\{\vec{m}, \ldots, \vec{m}^{L}\right\}$ with $|A|>\varepsilon L$ contain a geometric progression of length $k$, that is, there exist $\vec{a}, \vec{b} \in \mathbb{N}^{l}$ such that $\left\{\vec{a} \cdot \vec{b}, \ldots, \vec{a} \cdot \vec{b}^{k}\right\} \subset A$.

Proof Fix $k \in \mathbb{N}$ and $0<\varepsilon<1$. It follows from Theorem 2.1 that there is some subset $A^{\prime}$ of $\{1, \ldots, L\}$ with $\left|A^{\prime}\right|>\varepsilon L$ containing an arithmetic progression of length $k$, which implies that there exist $a, b \in \mathbb{N}$ such that $\{a+b, \ldots, a+k b\} \subset A^{\prime}$.

Now put $A=\left\{\vec{m}^{n}: n \in A^{\prime}\right\}$ and $\vec{a}=\vec{m}^{a}, \vec{b}=\vec{m}^{b}$. It is easy to see that such construction meets the requirement.

\subsection{Compact Metric Spaces}

In a compact metric space $(X, d)$, the open ball of radius $r$ centered at $x$ will be denoted by $B(x, r)$. The subset $B$ is $\varepsilon$-dense if for all $x \in X$, there exists $a \in B$ such that $d(x, a)<\varepsilon$.

For $A \subset X$ and $\delta>0$, let

$$
[A]_{\delta}=\{x \in X: \exists a \in A, d(x, a) \leq \delta\} .
$$

The set of all non-empty closed subsets of $X$ is defined by $\mathcal{F}(X)$. The Hausdorff metric, defined between $F, H \in \mathcal{F}(X)$ by

$$
d_{H}(F, H)=\inf \left\{\delta>0: F \subset[H]_{\delta} \text { and } H \subset[F]_{\delta}\right\},
$$

makes $\left(\mathcal{F}(X), d_{H}\right)$ a compact metric space.

The subset $A$ is residual if it contains a dense $G_{\delta}$ set. 


\subsection{Dynamical Systems}

Let $(X, G)$ be a topological dynamical system and $d$ a metric on $X$. The orbit of a point $x \in X$ under $G$ is the set $o_{G}(x)=\{g x: g \in G\}$ while that of a subset $Y \subset X$ is $o_{G}(Y)=\cup_{g \in G} g Y$. We denote the corresponding closures with $\bar{o}_{G}(x)$ and $\bar{o}_{G}(Y)$ respectively. The dynamical system $(X, G)$ is minimal if every point in $X$ has a dense orbit.

If $A$ is a non-empty closed subset of $X$ and $G A \subset A$, then $\left(A,\left.G\right|_{A}\right)$ is called a subsystem of $(X, G)$, where $\left.G\right|_{A}$ is the restriction of $G$ on $A$. If there is no ambiguity, we will use the notation $G$ instead of $\left.G\right|_{A}$.

It follows from Zorn's Lemma that any system contains a minimal subsystem.

Let $l \in \mathbb{N}$. Let $X$ be a compact metric space and let $T_{1}, \ldots, T_{l}: X \rightarrow X$ be $l$ commuting homeomorphisms of $X$, we write $\left(X, T_{1}, \ldots, T_{l}\right)$ to denote the dynamical system $\left(X,\left\langle T_{1}, \ldots, T_{l}\right\rangle\right)$, where $\left\langle T_{1}, \ldots, T_{l}\right\rangle$ is the group generated by $T_{1}, \ldots, T_{l}$. Throughout this paper those systems are called $\mathbb{Z}^{l}$-systems.

Let $\left(X, T_{1}, \ldots, T_{l}\right)$ be a $\mathbb{Z}^{l}$-system. Let $\vec{n}=\left(n_{1}, \ldots, n_{l}\right) \in \mathbb{Z}^{l}, x \in X$, for convenience we usually write $T_{1}^{n_{1}} \cdots T_{l}^{n_{l}} x$ as $T^{\vec{n}} x$. The set of return times of a point $x \in X$ to a non-empty open subset $U$ of $X$ is

$$
N(x, U)=\left\{\vec{n} \in \mathbb{Z}^{l}: T^{\vec{n}} x \in U\right\} .
$$

\subsection{Lower Semicontinuous Functions}

Let $X$ and $Y$ be compact metric spaces. A map $\varphi: X \rightarrow \mathcal{F}(Y)$ is lower semicontinuous (LSC) at $x \in X$ if for all $\varepsilon>0$, there exists $\delta>0$ such that for all $x^{\prime} \in X$ with $d\left(x, x^{\prime}\right)<\delta, \varphi(x) \subset\left[\varphi\left(x^{\prime}\right)\right]_{\varepsilon}$.

Lemma 2.3 Let $(X, G)$ be a dynamical system. The orbit closure map $\bar{o}_{G}: X \rightarrow$ $\mathcal{F}(X), x \mapsto \bar{o}_{G}(x)$ is LSC. In particular, it is Borel measurable, that is for any Borel subset $B \subset \mathcal{F}(X)$, the set $\bar{o}_{G}^{-1}(B) \subset X$ is Borel.

Proof Let $x \in X, \varepsilon>0$. As $\bar{o}_{G}(x)$ is compact, there exist $k \in \mathbb{N}$ and $g_{1}, \ldots, g_{k} \in G$ such that $\left\{g_{1} x, \ldots, g_{k} x\right\}$ is $\frac{\varepsilon}{2}$-dense in $\bar{o}_{G}(x)$. As each $g_{i}$ is continuous, there exists $\delta>0$ such that for all $x^{\prime} \in X$ with $d\left(x, x^{\prime}\right)<\delta, d\left(g_{i} x, g_{i} x^{\prime}\right)<\frac{\varepsilon}{2}$ for $i=1, \ldots, k$.

We next show that $\bar{o}_{G}(x) \subset\left[\bar{o}_{G}\left(x^{\prime}\right)\right]_{\varepsilon}$ for any $x^{\prime} \in B(x, \delta)$. Indeed, let $x^{\prime} \in B(x, \delta)$ and $y \in \bar{o}_{G}(x)$. Then there exists some $j \in\{1, \ldots, k\}$ with $d\left(g_{j} x, y\right)<\frac{\varepsilon}{2}$. So we have $d\left(g_{j} x^{\prime}, y\right) \leq d\left(g_{j} x^{\prime}, g_{j} x\right)+d\left(g_{j} x, y\right)<\varepsilon$, which implies $y \in\left[\bar{o}_{G}\left(x^{\prime}\right)\right]_{\varepsilon}$.

The second statement follows from the fact that when $X$ and $Y$ are compact metric spaces, all LSC functions $\varphi: X \rightarrow \mathcal{F}(Y)$ are Borel measurable (see [2, Lemma 17.5, Theorem 17.15, Theorem 18.10]).

\subsection{Invariant Measures}

For a dynamical system $(X, G)$ with $G$ abelian, we denote by $\mathcal{M}(X, G)$ the set of $G$-invariant Borel probability measures on $X$. It is well known that $\mathcal{M}(X, G)$ is non- 
empty. We regard $\mathcal{M}(X, G)$ as a closed convex subset of $C(X)^{*}$, equipped with the weak-* topology, then $\mathcal{M}(X, G)$ is a compact metric space.

Let $\mu \in \mathcal{M}(X, G)$. We define the support of $\mu$ by

$$
\operatorname{supp}(\mu)=\{x \in X: \mu(U)>0 \text { for any neighborhood } U \text { of } x\} .
$$

The action of $G$ on $X$ induces an action on $\mathcal{M}(X, G)$ in the following way: for $\mu \in \mathcal{M}(X, G)$ we define $g \mu$ by

$$
\int_{X} f(x) \mathrm{d}(g \mu)(x)=\int_{X} f(g x) \mathrm{d} \mu(x), \quad \forall f \in C(X) .
$$

Lemma 2.4 [9, Lemma 2.15] Let $X$ be a compact metric space. Suppose $\left\{\mu_{n}\right\}_{n \in \mathbb{N}}$ is a sequence of Borel probability measure converging in the weak-* topology to a probability measure $\mu$. If $\left\{H_{n}\right\}_{n \in \mathbb{N}}$ is a sequence of closed subsets of $X$ such that $\operatorname{supp}\left(\mu_{n}\right) \subset H_{n}$ and $H$ is a closed subset of $X$ such that $H_{n} \rightarrow H$ in the Hausdorff metric, then $\operatorname{supp}(\mu) \subset H$.

Fix a measure space $(X, \mathcal{B}, \mu)$ and $h \in \mathbb{N}$. We denote by $\mu_{\Delta_{h}}$ the diagonal measure on $X^{h}$ given by

$$
\int_{X^{h}} f\left(x_{1}, \ldots, x_{h}\right) \mathrm{d} \mu_{\Delta_{h}}=\int_{X} f(x, \ldots, x) \mathrm{d} \mu,
$$

where $f$ is a measurable function on $X^{h}$.

Notice that $\mu_{\Delta_{h}}\left(A_{1} \times \cdots \times A_{h}\right)=\mu\left(A_{1} \cap \cdots \cap A_{h}\right)$ for any $A_{1}, \ldots, A_{h} \in \mathcal{B}$.

\section{Decompositions of Minimal Systems}

In this section, inspired by Ye in [12], we obtain the following decomposition of minimal systems.

Theorem 3.1 Let $(X, G)$ be a minimal system. Let $H$ be a normal subgroup of $G$ with finite index $n$, then there exist closed sets $X_{i}, i=1, \ldots, n$, such that every $\left(X_{i}, H\right)$ is minimal and also we have the following decomposition:

$$
X=\bigcup_{i=1}^{n} X_{i}
$$

Proof Let $H$ be a normal subgroup of $G$ with finite index. Put $n=[G: H]$, then there exist $g_{1}, \ldots, g_{n} \in G$ such that $G$ is the mutually disjoint union of $g_{i} H, i=1, \ldots, n$. It is easy to see that $(X, H)$ is also a dynamical system. Let $X_{e}$ be a minimal subset of $X$ under $H$-action. For $g \in G$, put $X_{g}=g X_{e}$. Notice that $H$ is normal, we get $g H=H g$ which implies that $X_{g}$ is invariant under $H$-action. 
Claim For every $g \in G, X_{g}$ is minimal for $H$-action.

Proof of Claim Let $g \in G$. Let $Y_{g}$ be a closed subset of $X_{g}$ which is minimal for $H$-action. Then we have

$$
g^{-1} Y_{g} \subset g^{-1} X_{g}=g^{-1} g X_{e}=X_{e} .
$$

As $\left(X_{e}, H\right)$ is minimal and $g^{-1} Y_{g}$ is an $H$-invariant closed subset of $X_{e}$, we deduce that $g^{-1} Y_{g}=X_{e}$. Then

$$
X_{g}=g X_{e}=g\left(g^{-1} Y_{g}\right)=Y_{g}
$$

which implies $\left(X_{g}, H\right)$ itself is minimal.

It follows from the claim above that for any $g, g^{\prime} \in G, X_{g}=X_{g^{\prime}}$ or $X_{g} \cap X_{g^{\prime}}=\varnothing$. Moreover, for any $h \in H$, we have $X_{g}=X_{g h}=X_{h g}$.

Put $X_{0}=\cup_{i=1}^{n} X_{g_{i}}$, then $X_{0}$ is closed. We next show that $X_{0}$ is a $G$-invariant subset of $X$, which implies $X_{0}=X$ by the minimality of $(X, G)$.

Let $g \in G$. Recall that $H$ is normal, thus for any $i \in\{1, \ldots, n\}$, there is a unique $k_{i} \in\{1, \ldots, n\}$ such that $g g_{i} H=g_{k_{i}} H$. Let $h_{i} \in H$ with $g g_{i}=g_{k_{i}} h_{i}$. Now we have

$$
g X_{0}=\bigcup_{i=1}^{n} g X_{g_{i}}=\bigcup_{i=1}^{n} X_{g g_{i}}=\bigcup_{i=1}^{n} X_{g_{k_{i}} h_{i}}=\bigcup_{i=1}^{n} X_{g_{k_{i}}}=X_{0}
$$

which implies $X_{0}$ is $G$-invariant. We conclude that $X=\cup_{i=1}^{n} X_{g_{i}}$.

This completes the proof.

As a corollary of Theorem 3.1, we have:

Corollary 3.2 Let $\left(X, T_{1}, \ldots, T_{l}\right)$ be a minimal $\mathbb{Z}^{l}$-system. For any $\vec{n}=\left(n_{1}, \ldots, n_{l}\right) \in$ $\mathbb{Z}^{l}$ with $n_{1} \cdots n_{l} \neq 0$, there exist closed subsets $X_{\vec{i}}, \vec{i} \in[\vec{n}] \operatorname{such}$ that $\left(X_{\vec{i}}, T_{1}^{n_{1}}, \ldots, T_{l}^{n_{l}}\right)$ is minimal and also we have the following decomposition:

$$
X=\bigcup_{\vec{i} \in[\vec{n}]} X_{\vec{i}}
$$

Proof Let $\vec{n}=\left(n_{1}, \ldots, n_{l}\right) \in \mathbb{Z}^{l}$ with $n_{1} \cdots n_{l} \neq 0$. Put $H=\left\{T^{\vec{m} \cdot \vec{n}}: \vec{m} \in \mathbb{Z}^{l}\right\}$, then $H$ is a normal subgroup of $\left\langle T_{1}, \ldots, T_{l}\right\rangle$ with index $\left|n_{1} \cdots n_{l}\right|$ and $\left\langle T_{1}, \ldots, T_{l}\right\rangle=$ $\cup_{\vec{i} \in[\vec{n}]} T^{\vec{i}} H$. It follows from Theorem 3.1 that there are closed subsets $X_{\vec{i}}, \vec{i} \in[\vec{n}]$ such that every $\left(X_{\vec{i}}, T_{1}^{n_{1}}, \ldots, T_{l}^{n_{l}}\right)$ is minimal and also we have $X=\cup_{\vec{i} \in[\vec{n}]} X_{\vec{i}}$.

Lemma 3.3 Let $\left(X, T_{1}, \ldots, T_{l}\right)$ be a minimal $\mathbb{Z}^{l}$-system and $U$ be a non-empty open subset of $X$. There exists some $\vec{N} \in \mathbb{N}^{l}$ such that for all $\vec{n} \in \mathbb{Z}^{l}$ with $(\vec{N}, \vec{n})=\overrightarrow{1}$,

$$
\bigcup_{\vec{m} \in \mathbb{Z}^{l}} T^{\vec{m} \cdot \vec{n}} U=X
$$


Proof Let $U$ be a non-empty open subset of $X$. As $\left(X, T_{1}, \ldots, T_{l}\right)$ is minimal, there exist $k \in \mathbb{N}$ and $\vec{b}_{i} \in \mathbb{Z}^{l}, i=1, \ldots, k$ such that $X=\cup_{i=1}^{k} T^{\vec{b}_{i}} U$. Clearly, for any $\vec{b} \in \mathbb{Z}^{l}$, we still have $X=\cup_{i=1}^{k} T^{\left(\vec{b}+\vec{b}_{i}\right)} U$.

Claim The set

$$
B=\left\{\vec{n} \in \mathbb{N}^{l}: \bigcup_{\vec{m} \in \mathbb{Z}^{l}} T^{\vec{m} \cdot \vec{n}} U \neq X\right\}
$$

does not contain $k$ pairwise coprime elements.

Proof of Claim Let $\vec{n}=\left(n_{1}, \ldots, n_{l}\right) \in B$. By Corollary 3.2, there exist closed sets $X_{\vec{i}}, \vec{i} \in[\vec{n}]$ such that

$$
X=\bigcup_{\vec{i} \in[\vec{n}]} X_{\vec{i}}
$$

We show that there exists $\vec{j} \in[\vec{n}]$ such that $U \cap X_{\vec{j}}=\emptyset$. Otherwise for each $\vec{i} \in[\vec{n}]$, the set $U \cap X_{\vec{i}}$ is a non-empty open subset of $X_{\vec{i}}$. It follows from the minimality of the system $\left(X_{\vec{i}}, T_{1}^{n_{1}}, \ldots, T_{l}^{n_{l}}\right)$ that

$$
X=\bigcup_{\vec{i} \in[\vec{n}]} X_{\vec{i}}=\bigcup_{\vec{i} \in[\vec{n}]} \bigcup_{\vec{m} \in \mathbb{Z}^{l}} T^{\vec{m} \cdot \vec{n}}\left(U \cap X_{\vec{i}}\right)=\bigcup_{\vec{m} \in \mathbb{Z}^{l}} T^{\vec{m} \cdot \vec{n}} U,
$$

contradicting the fact that $\vec{n} \in B$.

We return to the proof of Claim.

Suppose for a contradiction that $\vec{n}_{1}, \ldots, \vec{n}_{k} \in B$ which are pairwise coprime. For every $i=1, \ldots, k$, by Corollary 3.2 , we have

$$
X=\bigcup_{\vec{j} \in\left[\vec{n}_{i}\right]} X_{\vec{j}}^{\left(\vec{n}_{i}\right)}
$$

and $T^{\vec{m}} X_{\vec{j}}^{\left(\vec{n}_{i}\right)}=X_{\vec{j}+\vec{m}}^{\left(\vec{n}_{i}\right)}=X_{\vec{a}}^{\left(\vec{n}_{i}\right)}$ if $\vec{j}+\vec{m} \equiv \vec{a} \bmod \vec{n}_{i}$. By the argument above, there exists $\vec{j}_{i} \in\left[\vec{n}_{i}\right], i=1, \ldots, k$ such that

$$
U \cap X_{\vec{j}_{i}}^{\left(\vec{n}_{i}\right)}=\emptyset
$$

As $\left\{X_{\vec{j}}^{\left(\vec{n}_{i}\right)}: \vec{j} \in\left[\vec{n}_{i}\right]\right\}$ covers $X$ for every $i$, there is $\vec{t}_{i} \in\left[\vec{n}_{i}\right]$ such that

$$
\bigcap_{i=1}^{k} X_{\vec{t}_{i}}^{\left(\vec{n}_{i}\right)} \neq \emptyset
$$


Recall that $\vec{n}_{1}, \ldots, \vec{n}_{k}$ are pairwise coprime, by Chinese Remainder Theorem, there exists some $\vec{a} \in \mathbb{Z}^{l}$ such that for every $i=1, \ldots, k$,

$$
\vec{a} \equiv \vec{j}_{i}+\vec{b}_{i}-\vec{t}_{i} \quad \bmod \vec{n}_{i}
$$

Then we have $X_{\vec{a}-\vec{b}_{i}+\vec{t}_{i}}^{\left(\vec{n}_{i}\right)}=X_{\vec{j}_{i}}^{\left(\vec{n}_{i}\right)}$ and

$$
\begin{aligned}
\bigcap_{i=1}^{k} X_{\vec{t}_{i}}^{\left(\vec{n}_{i}\right)} & \left.=\bigcup_{i=1}^{k} T^{\left(-\vec{a}+\vec{b}_{i}\right)} U\right) \cap \bigcap_{i=1}^{k} X_{\vec{t}_{i}}^{\left(\vec{n}_{i}\right)} \\
& \subset \bigcup_{i=1}^{k}\left(T^{\left(-\vec{a}+\vec{b}_{i}\right)} U\right) \cap X_{\vec{t}_{i}}^{\left(\vec{n}_{i}\right)} \\
& \subset \bigcup_{i=1}^{k} T^{\left(-\vec{a}+\vec{b}_{i}\right)}\left(U \cap T^{\left(\vec{a}-\vec{b}_{i}\right)} X_{\vec{t}_{i}}^{\left(\vec{n}_{i}\right)}\right) \\
& \subset \bigcup_{i=1}^{k} T^{\left(-\vec{a}+\vec{b}_{i}\right)}\left(U \cap X_{\vec{a}-\vec{b}_{i}+\vec{t}_{i}}^{\left(\vec{n}_{i}\right)}\right. \\
& =\bigcup_{i=1}^{k} T^{\left(-\vec{a}+\vec{b}_{i}\right)}\left(U \cap X_{\vec{j}_{i}}^{\left(\vec{n}_{i}\right)}\right)=\emptyset \text { by (3.1), }
\end{aligned}
$$

contradicting (3.2). This completes the proof.

Using the claim above, we can easily get the result. Indeed, let $d$ be the maximal number such that there exist $d$ pairwise coprime elements. Then $d<k$. If $d=1$, this means any two elements in $B$ are not coprime. Choose any $\vec{a} \in B$ and put $\vec{N}=\vec{a}$. If $d>1$, choose $\vec{a}_{1}, \ldots, \vec{a}_{d} \in B$ such that they are pairwise coprime and put $\vec{N}=\vec{a}_{1} \cdots \vec{a}_{d}$. Clearly, the choice of $\vec{N}$ meets the requirement.

This completes the proof.

Proposition 3.4 Let $\left(X, T_{1}, \ldots, T_{l}\right)$ be a minimal $\mathbb{Z}^{l}$-system, then for every $\varepsilon>0$, there exists some $\vec{N} \in \mathbb{N}^{l}$, such that for all $\vec{n} \in \mathbb{Z}^{l}$ with $(\vec{n}, \vec{N})=\overrightarrow{1}$ and $x \in X$, the set

$$
\left\{T^{\vec{m} \cdot \vec{n}} x: \vec{m} \in \mathbb{Z}^{l}\right\}
$$

is $\varepsilon$-dense in $X$.

Proof Fix $\varepsilon>0$. Since $X$ is compact, there exist $n \in \mathbb{N}$ and points $x_{1}, \ldots, x_{n} \in X$ such that $X=\cup_{j=1}^{n} B\left(x_{j}, \frac{\varepsilon}{2}\right)$. For every $j \in\{1, \ldots, n\}$, by Lemma 3.3 there exists some $\vec{N}_{j} \in \mathbb{N}^{l}$ such that for all $\vec{n} \in \mathbb{Z}^{l}$ with $\left(\vec{n}, \vec{N}_{j}\right)=\overrightarrow{1}$, we have

$$
\bigcup_{\vec{m} \in \mathbb{Z}^{l}} T^{-\vec{m} \cdot \vec{n}} B\left(x_{j}, \frac{\varepsilon}{2}\right)=X .
$$


Let $\vec{N}=\vec{N}_{1} \cdots \vec{N}_{n}$, we next show that $\vec{N}$ has the required property.

Let $\vec{n} \in \mathbb{Z}^{l}$ with $(\vec{n}, \vec{N})=\overrightarrow{1}$ and $x \in X$. For every $z \in X$, there exists some $j \in\{1, \ldots, n\}$ with $d\left(z, x_{j}\right)<\frac{\varepsilon}{2}$. For such $j$, by (3.3), there is $\vec{m} \in \mathbb{Z}^{l}$ such that $T^{\vec{m} \cdot \vec{n}} x \in B\left(x_{j}, \frac{\varepsilon}{2}\right)$. Thus

$$
d\left(T^{\vec{m} \cdot \vec{n}} x, z\right) \leq d\left(T^{\vec{m} \cdot \vec{n}} x, x_{j}\right)+d\left(x_{j}, z\right)<\varepsilon .
$$

This completes the proof.

\section{Dynamics on the Orbit Closure of the Diagonal}

In this section, we will show our main result. Before proving let us say something about the idea in the proof. Let $h, l \in \mathbb{N}$. Let $\left(X, T_{1}, \ldots, T_{l}\right)$ be a minimal $\mathbb{Z}^{l}$-system and let $x \in X, U$ be a non-empty open subset of $X$. Finding a configuration of the form $\left\{\vec{n} \cdot \vec{m}, \ldots, \vec{n} \cdot \vec{m}^{h}\right\}$ in $N(x, U)$ is equivalent to showing that the $T^{\vec{n} \cdot \vec{m}} \times \cdots \times T^{\vec{n} \cdot \vec{m}^{h}}-$ orbit closure of $(x, \ldots, x)$ in $X^{h}$ has non-empty intersection with $U \times \cdots \times U$. To this, following the idea in [9], we first study the dynamics of points along the diagonal of $X^{h}$ under $T^{\vec{m}_{1}} \times \cdots \times T^{\vec{m}_{h}}$-action, where $\vec{m}_{1}, \ldots, \vec{m}_{h}$ are all prime to $\vec{N}$ depend on $U$ by Lemma 3.3.

Precisely, let $\vec{m}_{k}=\left(m_{1}^{(k)}, \ldots, m_{l}^{(k)}\right) \in \mathbb{N}^{l}, k=1, \ldots, h$. Define a $\mathbb{Z}^{l}$-system from $X^{h}$ to itself via the map:

$$
S_{i}\left(x_{1}, \ldots, x_{h}\right)=\left(T_{i}^{m_{i}^{(1)}} x_{1}, \ldots, T_{i}^{m_{i}^{(h)}} x_{h}\right), \quad i=1, \ldots, l .
$$

Put $\mathbf{m}=\left(\vec{m}_{1}, \ldots, \vec{m}_{h}\right)$. For $\vec{n} \in \mathbb{Z}^{l}$, let $T^{\vec{n} \cdot \mathbf{m}}=\left(T^{\vec{n} \cdot \vec{m}_{1}}, \ldots, T^{\vec{n} \cdot \vec{m}_{h}}\right)$, then

$$
S_{1}^{n_{1}} \ldots S_{l}^{n_{l}}\left(x_{1}, \ldots, x_{h}\right)=\left(T^{\vec{n} \cdot \vec{m}_{1}} x_{1}, \ldots, T^{\vec{n} \cdot \vec{m}_{h}} x_{h}\right)=T^{\vec{n} \cdot \mathbf{m}}\left(x_{1}, \ldots, x_{h}\right) .
$$

Let $\left\langle T^{\mathbf{m}}\right\rangle$ be the group generated by $\left\{T^{\vec{n} \cdot \mathbf{m}}=\left(T^{\vec{n} \cdot \vec{m}_{1}}, \ldots, T^{\vec{n} \cdot \vec{m}_{h}}\right): \vec{n} \in \mathbb{Z}^{l}\right\}$. We denote the system $\left(X^{h}, S_{1}, \ldots, S_{l}\right)$ by $\left(X^{h}, T^{\mathbf{m}}\right)$ and the orbit of $\vec{x} \in X^{h}$ by $o_{\mathbf{m}}(\vec{x})=$ $\left\{T^{\vec{n} \cdot \mathbf{m}} \vec{x}: \vec{n} \in \mathbb{Z}^{l}\right\}$. Let $\Delta: X \rightarrow X^{h}, x \mapsto(x, \ldots, x)$ and let

$$
\begin{aligned}
& \Delta\left(T^{\vec{n}}\right)=T^{\vec{n}} \times \cdots \times T^{\vec{n}} \quad(h \text { times }),
\end{aligned}
$$

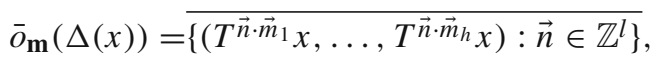

$$
\begin{aligned}
& X^{\Delta}=\bar{o}_{\mathbf{m}}(\Delta(X))=\overline{\left\{\left(T^{\vec{n} \cdot \vec{m}_{1}} x, \ldots, T^{\vec{n} \cdot \vec{m}_{h}} x\right): x \in X, \vec{n} \in \mathbb{Z}^{l}\right\} .}
\end{aligned}
$$

Note that for any $\vec{n} \in \mathbb{Z}^{l}, \Delta\left(T^{\vec{n}}\right)$ is a homeomorphism of $X^{h}$.

We will prove some preliminary results concerning dynamics of points along the diagonal of $X^{h}$ and points of continuity of the orbit closure map $\bar{o}_{\mathbf{m}}$.

Let $\vec{M}=\operatorname{LCM}\left(\vec{m}_{1}, \ldots, \vec{m}_{h}\right)=\left(M_{1}, \ldots, M_{l}\right) \in \mathbb{N}^{l}$. By Corollary 3.2 , we have the decomposition: $X=\cup_{\vec{i} \in[\vec{M}]} X_{\vec{i}}$. 
Proposition 4.1 Let $\vec{j} \in[\vec{M}]$ and let $U$ be an open subset of $X$ with $X_{\vec{j}} \cap U \neq \emptyset$, then the set

$$
X_{U}^{\Delta}=\bar{o}_{\mathbf{m}}\left(\Delta\left(X_{\vec{j}} \cap U\right)\right)
$$

has non-empty interior in $X_{\vec{j}}^{\Delta}=\bar{o}_{\mathbf{m}}\left(\Delta\left(X_{\vec{j}}\right)\right)$.

Proof By Corollary 3.2, the system $\left(X_{\vec{j}}, T^{M_{1}}, \ldots, T^{M_{l}}\right)$ is minimal, then there exist $t \in \mathbb{N}$ and $\vec{b}_{i} \in \mathbb{Z}^{l}, i=1, \ldots, t$ such that $\cup_{i=1}^{t} T^{\vec{b}_{i} \cdot \vec{M}}\left(X_{\vec{j}} \cap U\right)=X_{\vec{j}}$. Notice that

$$
X_{\vec{j}}^{\Delta}=\bar{o}_{\mathbf{m}}\left(\Delta\left(X_{\vec{j}}\right)\right)=\bar{o}_{\mathbf{m}}\left(\Delta\left(\bigcup_{i=1}^{t} T^{\overrightarrow{b_{i}} \cdot \vec{M}}\left(X_{\vec{j}} \cap U\right)\right)\right)=\bigcup_{i=1}^{t} \Delta\left(T^{\overrightarrow{b_{i}} \cdot \vec{M}}\right) X_{U}^{\Delta}
$$

Since $X_{\vec{j}}^{\Delta}$ is a Baire space, there is some $i \in\{1, \ldots, t\}$ such that $\Delta\left(T^{\overrightarrow{b_{i}} \cdot \vec{M}}\right) X_{U}^{\Delta}$ has non-empty interior. As $\Delta\left(T^{\overrightarrow{b_{i}} \cdot \vec{M}}\right)$ is homeomorphic, it is open, implying that $X_{U}^{\Delta}$ has non-empty interior.

The following result shows that for any dynamical system, the points of discontinuity of the orbit closure map can be covered by countably many closed invariant subsets with empty interior.

Lemma 4.2 Let $(X, G)$ be a dynamical system and denote by $\Lambda$ the set of points of discontinuity of the map $\bar{o}_{G}: X \rightarrow \mathcal{F}(X)$. There is a countable family $\left\{B_{i}\right\}_{i \in \mathbb{N}}$ of closed, G-invariant, empty interior subsets of $X$ such that $\Lambda \subset \cup_{i \in \mathbb{N}} B_{i}$.

Proof For $A \in \mathcal{F}(X)$ and $\varepsilon>0, \delta>0$, let

$$
M(A, \varepsilon)=\max \left\{n \in \mathbb{N}: \exists a_{1}, \ldots, a_{n} \in A, \text { s.t. } d\left(a_{i}, a_{j}\right)>\varepsilon, \forall i \neq j\right\},
$$

and let

$$
U(A, \delta)=\{x \in X: \exists a \in A, d(x, a)<\delta\}
$$

For $n \in \mathbb{N}$, let

$$
B_{n, \varepsilon}=\left\{x \in X: \begin{array}{c}
M\left(\bar{o}_{G}(x), \varepsilon\right) \leq n, \text { and } \\
\forall \varepsilon^{\prime} \in(0,3 \varepsilon), \forall U \ni x(\text { open }), \exists y \in U, \bar{o}_{G}(y) \not \subset U\left(\bar{o}_{G}(x), \varepsilon^{\prime}\right)
\end{array}\right\} .
$$

It is proved in [7, Theorem 1], that each $B_{n, \varepsilon}$ is closed with empty interior and

$$
\Lambda \subset \bigcup\left\{B_{n, \varepsilon}: n \in \mathbb{N}, \varepsilon \in \mathbb{Q}_{+}\right\}
$$

It is clear that every $B_{n, \varepsilon}$ is $G$-invariant as $\bar{o}_{G}(g x)=\bar{o}_{G}(x)$ for any $g \in G$ and $x \in X$.

This completes the proof. 
By Proposition 4.1 and Lemma 4.2, we will show that for system $\left(X^{h}, T^{\mathbf{m}}\right)$, points of continuity of the map $\bar{o}_{\mathbf{m}}$ have non-empty intersection with the diagonal of $X^{h}$ which are also $\left\langle T^{\mathbf{m}}\right\rangle$-invariant. In particular, if $\Delta(x)$ is the point of continuity of the map $\bar{o}_{\mathbf{m}}$ for some $x \in X$, so is $\Delta\left(T^{\vec{n} \cdot \mathbf{m}} x\right)$ for any $\vec{n} \in \mathbb{Z}^{l}$. This fact is the key part in the proof of Proposition 4.5.

Proposition 4.3 Let $\Omega$ be the set of points of continuity of the map $\bar{o}_{\mathbf{m}}: X^{\Delta} \rightarrow \mathcal{F}\left(X^{\Delta}\right)$. The set $\Delta(X) \cap \Omega$ is a residual subset of $\Delta(X)$.

Proof Let $\Lambda=X^{\Delta} \backslash \Omega$ be the set of points of discontinuity of the map $\bar{o}_{\mathbf{m}}: X^{\Delta} \rightarrow$ $\mathcal{F}\left(X^{\Delta}\right)$. By Lemma 4.2 there is a countable family $\left\{B_{i}\right\}_{i \in \mathbb{N}}$ of closed, $\left\langle T^{\mathbf{m}}\right\rangle$-invariant, empty interior subsets of $X^{\Delta}$ such that $\Lambda \subset \cup_{i \in \mathbb{N}} B_{i}$.

We claim that each $\Delta(X) \cap B_{i}$ is a closed set with empty interior in $\Delta(X)$. Clearly, it is closed since $\Delta(X)$ is closed. Suppose for a contradiction that there is some open subset $U$ of $X$ such that $\Delta(U) \subset B_{i}$. Then we have $X_{U}^{\Delta}=\bar{o}_{\mathbf{m}}(\Delta(U)) \subset B_{i}$ since $B_{i}$ is $\left\langle T^{\mathbf{m}}\right\rangle$-invariant and closed. It follows by Proposition 4.1 that $B_{i}$ has non-empty interior, a contradiction.

Note that $\Delta(X) \cap \Lambda \subset \cup_{i \in \mathbb{N}}\left(\Delta(X) \cap B_{i}\right)$ is a cover of $\Delta(X) \cap \Lambda$ with closed sets with empty interior.

Since $\Delta(X)=(\Delta(X) \cap \Omega) \cup(\Delta(X) \cap \Lambda)$, the set $\Delta(X) \cap \Omega$ is a residual subset of $\Delta(X)$.

We use the method introduced in [10] to show the following result and we postpone it to "Appendix A".

Theorem 4.4 Let $\left(X, T_{1}, \ldots, T_{l}\right)$ be a minimal $\mathbb{Z}^{l}$-system. For given $\vec{m}_{1}, \ldots, \vec{m}_{h} \in \mathbb{Z}^{l}$, let $\mathbf{m}=\left(\vec{m}_{1}, \ldots, \vec{m}_{h}\right)$ and let $X^{\Delta}=\bar{o}_{\mathbf{m}}(\Delta(X))$, then $\left(X^{\Delta}, G\right)$ is minimal, where $G$ is the group generated by $\left\{T^{\Delta(\vec{n})+\vec{m} \cdot \mathbf{m}}=\left(T^{\vec{n}+\vec{m} \cdot \vec{m}_{1}}, \ldots, T^{\vec{n}+\vec{m} \cdot \vec{m}_{h}}\right): \vec{n}, \vec{m} \in \mathbb{Z}^{l}\right\}$.

Proposition 4.5 Let $\left(X, T_{1}, \ldots, T_{l}\right)$ be a minimal $\mathbb{Z}^{l}$-system and let $U$ be a non-empty open subset of $X$. There exists $\eta>0$ such that for all $\varepsilon>0$, there exists $\vec{N} \in \mathbb{N}^{l}$ such that for any finite set $F \subset S_{\vec{N}} \cap \mathbb{N}^{l}$, there exists an $\varepsilon$-dense subset $X_{\varepsilon} \subset X$ such that for all $x \in X_{\varepsilon}$, there exists $F^{\prime} \subset F$ with $\left|F^{\prime}\right|>\eta|F|$ and $\vec{n} \in \mathbb{N}^{l}$ such that $\vec{n} \cdot F^{\prime} \subset N(x, U)$.

Proof Let $V$ be a non-empty open subset of $X$ such that $\bar{V} \subset U$, and let $\mu$ be any $\left\langle T_{1}, \ldots, T_{l}\right\rangle$-invariant probability measure on $X$. Since $\left(X, T_{1}, \ldots, T_{l}\right)$ is minimal, we have $\eta=\left(\frac{\mu(V)}{2}\right)^{2}>0$. Let $\varepsilon>0$ and let $\vec{N}$ be as given in Proposition 3.4.

Let $F=\left\{\vec{m}_{1}, \ldots, \vec{m}_{h}\right\} \subset S_{\vec{N}} \cap \mathbb{N}^{l}$ and put $\mathbf{m}=\left(\vec{m}_{1}, \ldots, \vec{m}_{h}\right)$. Let

$$
X^{\Delta}=\bar{o}_{\mathbf{m}}(\Delta(X)) \text {. }
$$

Let $\mu^{\Delta}$ be any weak-* limit point of the set

$$
\left\{\frac{1}{\prod_{i=1}^{l} N_{i}} \sum_{\substack{\vec{n}=\left(n_{1}, \ldots, n_{l}\right) \\ 1 \leq n_{i} \leq N_{i}, i=1, \ldots, l}} T^{\vec{n} \cdot \mathbf{m}} \mu_{\Delta_{h}}: \vec{N}=\left(N_{1}, \ldots, N_{l}\right) \in \mathbb{N}^{l}\right\} .
$$


Then $\mu^{\Delta}$ is a $\left\langle T^{\mathbf{m}}\right\rangle$-invariant probability measure on $X^{\Delta}$ with $\pi_{i} \mu^{\Delta}=\mu$, where $\pi_{i}: X^{\Delta} \rightarrow X$ is the projection onto the $i^{\text {th }}$ coordinate.

By Lemma 2.3, the map $\bar{o}_{\mathbf{m}}: X^{\Delta} \rightarrow \mathcal{F}\left(X^{\Delta}\right)$ is LSC, hence Borel. Denote by $\mathcal{B}_{X^{\Delta}}$ and $\mathcal{B}_{\mathcal{F}\left(X^{\Delta}\right)}$ the Borel sigma-algebras of $X^{\Delta}$ and $\mathcal{F}\left(X^{\Delta}\right)$ respectively. Let $\mathcal{A}$ be the pull back of $\mathcal{B}_{\mathcal{F}\left(X^{\Delta}\right)}$ through $\bar{o}_{\mathbf{m}}$. As $\bar{o}_{\mathbf{m}}$ is Borel, $\mathcal{A}$ is a sub-sigma-algebra of $\mathcal{B}_{X^{\Delta}}$. Moreover $\mathcal{B}_{\mathcal{F}\left(X^{\Delta}\right)}$ is countably generated, so is $\mathcal{A}$.

For $\vec{x} \in X^{\Delta}$, let $\alpha(\vec{x})=\bar{o}_{\mathbf{m}}^{-1}\left\{\bar{o}_{\mathbf{m}}(\vec{x})\right\}$, then $\alpha(\vec{x})$ is the atom of $\mathcal{A}$ containing $\vec{x}$. Disintegrating $\mu^{\Delta}$ with respect to $\mathcal{A}$ (see [5, Theorem 5.14]), there exists a $\mu^{\Delta}$-conull set $X_{0}^{\Delta} \subset X^{\Delta}$ and for every $\vec{x} \in X_{0}^{\Delta}$, a Borel probability measure $\mu_{\vec{x}}^{\Delta}$ supported on $\alpha(\vec{x})$ such that $\mu_{\vec{x}}^{\Delta}=\mu_{\vec{y}}^{\Delta}$ whenever $\alpha(\vec{x})=\alpha(\vec{y})$ and

$$
\mu^{\Delta}=\int \mu_{\vec{x}}^{\Delta} \mathrm{d} \mu^{\Delta} .
$$

Notice that if $\vec{x}$ is a $\left\langle T^{\mathbf{m}}\right\rangle$-recurrent point, then $\bar{o}_{\mathbf{m}}(\vec{x})=\bar{o}_{\mathbf{m}}\left(T^{\vec{n} \cdot \mathbf{m}} \vec{x}\right)$ and $\alpha(\vec{x})=$ $\alpha\left(T^{\vec{n} \cdot \mathbf{m}} \vec{x}\right)$ for any $\vec{n} \in \mathbb{Z}^{l}$ which implies that $\mu_{\vec{x}}^{\Delta}$ is $\left\langle T^{\mathbf{m}}\right\rangle$-invariant. By [3, Proposition 4.2.2], the $\left\langle T^{\mathbf{m}}\right\rangle$-recurrent points of $X^{\Delta}$ have full measure. So we may assume that for all $\vec{x} \in X_{0}^{\Delta}$, the measure $\mu_{\vec{x}}^{\Delta}$ is $\left\langle T^{\mathbf{m}}\right\rangle$-invariant.

For $i \in\{1, \ldots, h\}$, let

$$
X_{i}^{\Delta}=\left\{\vec{x} \in X^{\Delta}:\left(\pi_{i} \mu_{\vec{x}}^{\Delta}\right)(\bar{V})>\sqrt{\eta}\right\} .
$$

Since

$$
\begin{aligned}
2 \sqrt{\eta} \leq \mu(\bar{V})=\left(\pi_{i} \mu^{\Delta}\right)(\bar{V}) & =\mu^{\Delta}\left(\pi_{i}^{-1}(\bar{V})\right) \\
& =\int \mu_{\vec{x}}^{\Delta}\left(\pi_{i}^{-1}(\bar{V})\right) \mathrm{d} \mu^{\Delta} \\
& =\int\left(\pi_{i} \mu_{\vec{x}}^{\Delta}\right)(\bar{V}) \mathrm{d} \mu^{\Delta} \\
& =\int_{X_{i}^{\Delta}}\left(\pi_{i} \mu_{\vec{x}}^{\Delta}\right)(\bar{V}) \mathrm{d} \mu^{\Delta}+\int_{X^{\Delta} \backslash X_{i}^{\Delta}}\left(\pi_{i} \mu_{\vec{x}}^{\Delta}\right)(\bar{V}) \mathrm{d} \mu^{\Delta} \\
& \leq \mu^{\Delta}\left(X_{i}^{\Delta}\right)+\sqrt{\eta}\left(1-\mu^{\Delta}\left(X_{i}^{\Delta}\right)\right),
\end{aligned}
$$

we have that $\mu^{\Delta}\left(X_{i}^{\Delta}\right)>\sqrt{\eta}$. By the pigeonhole principle, there exists $I \subset\{1, \ldots, h\}$ with $|I|>\sqrt{\eta} h$ such that $X_{0}^{\Delta} \cap\left(\bigcap_{i \in I} X_{i}^{\Delta}\right) \neq \emptyset$. Let $\vec{w}$ be an element of this set.

Put $\vec{M}=\operatorname{LCM}\left(\vec{m}_{1}, \ldots, \vec{m}_{h}\right)$. By Proposition 4.3 , there exists some point $x \in X$ such that $\vec{x}=\Delta(x)$ is a point of continuity of the map $\bar{o}_{\mathbf{m}}$. By Theorem 4.4 , there exist sequences $\left\{\vec{n}_{j}\right\}_{j \in \mathbb{N}},\left\{\vec{s}_{j}\right\}_{j \in \mathbb{N}} \subset \mathbb{Z}^{l}$ such that

$$
T^{\vec{n}_{j} \cdot \mathbf{m}+\Delta\left(\vec{s}_{j}\right)} \vec{w} \rightarrow \vec{x}, \quad j \rightarrow \infty
$$

By passing to a subsequence if necessary, there is some $\vec{b} \in[\vec{M}]$ such that for all $j \in \mathbb{N}, \vec{s}_{j} \equiv \vec{b} \bmod \vec{M}$. By Proposition 4.3 , the points of continuity of the map 
$\bar{o}_{\mathbf{m}}$ are $\Delta\left(T^{\vec{n}}\right)$-invariant for any $\vec{n} \in \mathbb{Z}^{l}$. Therefore, by replacing $x$ with $T^{-\vec{b}} x$ and sequence $\left\{\vec{s}_{j}\right\}_{j \in \mathbb{N}}$ with $\left\{\vec{s}_{j}-\vec{b}\right\}_{j \in \mathbb{N}}$, we may assume that $\vec{b}=\overrightarrow{0}$, that is $\left\{\vec{s}_{j}\right\}_{j \in \mathbb{N}} \subset$ $\langle\vec{M}\rangle=\left\{\vec{n} \cdot \vec{M}: \vec{n} \in \mathbb{Z}^{l}\right\}$.

Put $X_{\varepsilon}=\left\{T^{\vec{k} \cdot \vec{M}} x: \vec{k} \in \mathbb{Z}^{l}\right\}$. Since $\vec{M} \in S_{\vec{N}}$, by Proposition $3.4, X_{\varepsilon}$ is $\varepsilon$-dense in $X$. It is sufficient to show that for every $\vec{k} \in \mathbb{Z}^{l}$, there exists $F^{\prime} \subset F$ with $\left|F^{\prime}\right|>\eta|F|$ and $\vec{n} \in \mathbb{Z}^{l}$ such that $\vec{n} \cdot F^{\prime} \subset N\left(T^{\vec{k} \cdot \vec{M}} x, U\right)$.

Case $\vec{k}=\overrightarrow{0}$.

Proof of Case $\vec{k}=\overrightarrow{0}$

Let $v_{x}^{\Delta}$ be a weak-* limit point of the set $\left\{\Delta\left(T^{\vec{s}_{j}}\right) \mu_{\vec{w}}^{\Delta}: j \in \mathbb{N}\right\}$, we may assume without loss of generality that $\Delta\left(T^{\vec{s}_{j}}\right) \mu_{\vec{w}}^{\Delta} \rightarrow v_{x}^{\Delta}, j \rightarrow \infty$. We claim that $v_{x}^{\Delta}$ is a $\left\langle T^{\mathbf{m}}\right\rangle$-invariant probability measure supported on $x^{\Delta}=\bar{o}_{\mathbf{m}}(\vec{x})$ such that for all $i \in I,\left(\pi_{i} v_{x}^{\Delta}\right)(\bar{V})>\sqrt{\eta}$.

By definition, $v_{x}^{\Delta}$ is a probability measure. Since every element of $\left\langle T^{\mathbf{m}}\right\rangle$ commutes with $\Delta\left(T^{\vec{s}_{j}}\right)$ and $\mu_{\vec{w}}^{\Delta}$ is $\left\langle T^{\mathbf{m}}\right\rangle$-invariant, we deduce that $v_{x}^{\Delta}$ is $\left\langle T^{\mathbf{m}}\right\rangle$-invariant. As $\vec{x}$ is a point of continuity of $\bar{o}_{\mathbf{m}}$ and by (4.1), we obtain that

$$
\bar{o}_{\mathbf{m}}\left(T^{\vec{n}_{j} \cdot \mathbf{m}+\Delta\left(\vec{s}_{j}\right)} \vec{w}\right) \rightarrow \bar{o}_{\mathbf{m}}(\vec{x})=x^{\Delta}, \quad j \rightarrow \infty .
$$

It follows from Lemma 2.4 that the measure $v_{x}^{\Delta}$ is supported on $x^{\Delta}$.

Let $i \in I$. As $\mu_{\vec{w}}^{\Delta}$ is $\left\langle T^{\mathbf{m}}\right\rangle$-invariant, the measure $\pi_{i} \mu_{\vec{w}}^{\Delta}$ is $T^{\vec{n} \cdot \vec{m}_{i}}$-invariant for any $\vec{n} \in \mathbb{Z}^{l}$, hence $T^{\vec{s}_{j}}$-invariant for all $j \in \mathbb{N}$. Then we have

$$
\begin{aligned}
\left(\pi_{i} v_{x}^{\Delta}\right)(\bar{V}) & =v_{x}^{\Delta}\left(\pi_{i}^{-1}(\bar{V})\right) \\
& \geq \limsup _{j \rightarrow \infty}\left(\Delta\left(T^{\vec{s}_{j}}\right) \mu_{\vec{w}}^{\Delta}\right)\left(\pi_{i}^{-1}(\bar{V})\right) \\
& =\limsup _{j \rightarrow \infty}\left(T^{\vec{s}_{j}} \pi_{i} \mu_{\vec{w}}^{\Delta}\right)(\bar{V}) \\
& =\left(\pi_{i} \mu_{\vec{w}}^{\Delta}\right)(\bar{V})>\sqrt{\eta} .
\end{aligned}
$$

Let $g$ be a function defined on $x^{\Delta}$ by $g(\vec{y})=\frac{1}{h} \sum_{i=1}^{h} \mathbf{1}_{\bar{V}}\left(\pi_{i} \vec{y}\right), \vec{y} \in x^{\Delta}$, where $\mathbf{1}_{\bar{V}}$ is the character function of $\bar{V}$. Now, we have

$$
\begin{aligned}
\int_{x^{\Delta}} g(\vec{y}) \mathrm{d} v_{x}^{\Delta} & =\int_{x^{\Delta}} \frac{1}{h} \sum_{i=1}^{h} \mathbf{1}_{\bar{V}}\left(\pi_{i} \vec{y}\right) \mathrm{d} v_{x}^{\Delta} \\
& \geq \frac{1}{h} \sum_{i \in I} \int_{x^{\Delta}} \mathbf{1}_{\bar{V}}\left(\pi_{i} \vec{y}\right) \mathrm{d} v_{x}^{\Delta} \\
& =\frac{1}{h} \sum_{i \in I}\left(\pi_{i} v_{x}^{\Delta}\right)(\bar{V})>\frac{|I|}{h} \sqrt{\eta}>\eta .
\end{aligned}
$$

There is some point $\vec{z} \in x^{\Delta}$ with $g(\vec{z})>\eta$, that is,

$$
A=\left\{i \in\{1, \ldots, h\}: \pi_{i} \vec{z} \in \bar{V}\right\} \text { and }|A|>h \eta .
$$


As $\vec{z} \in x^{\Delta}=\bar{o}_{\mathbf{m}}(\Delta(x))$, there exists some $\vec{n} \in \mathbb{N}^{l}$ such that $T^{\vec{n} \cdot \vec{m}_{i}} x \in U, i \in A$.

Put $F^{\prime}=\left\{\vec{m}_{i}: i \in A\right\}$, then $\left|F^{\prime}\right|=|A|>\eta|F|$, and for every $i \in A$, we have $T^{\vec{n} \cdot \vec{m}_{i}} x \in U$. This completes the proof of Case $\vec{k}=\overrightarrow{0}$.

General cases Fix $\vec{k} \in \mathbb{Z}^{l}$. Then we have $\left\{\vec{s}_{j}+\vec{k} \cdot \vec{M}\right\}_{j \in \mathbb{N}} \subset\langle\vec{M}\rangle$ and

$$
T^{\vec{n}_{j} \cdot \mathbf{m}+\Delta\left(\vec{s}_{j}+\vec{k} \cdot \vec{M}\right)} \vec{w} \rightarrow \Delta\left(T^{\vec{k} \cdot \vec{M}} x\right), \quad j \rightarrow \infty .
$$

Notice that $\Delta\left(T^{\vec{k} \cdot \vec{M}} x\right)$ is still the point of continuity of the map $\bar{o}_{\mathbf{m}}$. By replacing $x$ with $T^{\vec{k} \cdot \vec{M}} x$ and $\left\{\vec{s}_{j}\right\}_{j \in \mathbb{N}}$ with $\left\{\vec{s}_{j}+\vec{k} \cdot \vec{M}\right\}_{j \in \mathbb{N}}$, and by the argument for case $\vec{k}=\overrightarrow{0}$, we deduce that the result also holds for general cases.

This completes the proof.

Theorem 4.6 Let $\left(X, T_{1}, \ldots, T_{l}\right)$ be a minimal $\mathbb{Z}^{l}$-system. Then there exists a residual subset $X^{\prime}$ such that for any $x \in X^{\prime}$ and any non-empty open subset $U$ of $X$ such that the set $N(x, U)$ contains arbitrarily long geometric progressions in $\mathbb{N}^{l}$.

Proof By taking a countable basis of open set and a countable intersection of residual sets, it is sufficient to show there is a residual set $X^{\prime}$ for the fixed open subset.

Let $U$ be an open non-empty subset of $X$. For $k \in \mathbb{N}$, put

$$
W_{k}=\bigcup_{\vec{m}, \vec{n} \in \mathbb{N}^{l}}\left(T^{-\vec{n} \cdot \vec{m}} U\right) \cap \cdots \cap\left(T^{-\vec{n} \cdot \vec{m}^{k}} U\right) .
$$

Then for every $x \in W_{k}, N(x, U)$ contains a geometric progression of length $k$ in $\mathbb{N}^{l}$. Put $X^{\prime}=\cap_{k \in \mathbb{N}} W_{k}$. Then for every $x \in X^{\prime}, N(x, U)$ contains arbitrarily long geometric progressions in $\mathbb{N}^{l}$.

We will show that $X^{\prime}$ is residual by showing that each $W_{k}$ is open and dense in $X$. As $W_{k}$ is open by definition, we have only to show that $W_{k}$ is $\varepsilon$-dense in $X$ for any $\varepsilon>0$.

Let $\eta>0$ be as given in Proposition 4.5 for the set $U$. Fix $k \in \mathbb{N}$ and $\varepsilon>0$. Let $\vec{N}$ be as given in Proposition 4.5. By Proposition 2.2, there exists $K \in \mathbb{N}$ such that any $\eta$-dense subset of any geometric progression of length $K$ contains a geometric progression of length $k$. Let $F \subset S_{\vec{N}} \cap \mathbb{N}^{l}$ be a geometric progression of length $K$. Let $X_{\varepsilon} \subset X$ be as given in Proposition 4.5. To show that $W_{k}$ is $\varepsilon$-dense, it suffices to show that $X_{\varepsilon} \subset W_{k}$.

Let $x \in X_{\varepsilon}$. By Proposition 4.5, there exists $F^{\prime} \subset F$ with $\left|F^{\prime}\right|>\eta|F|$ and $\vec{n} \in \mathbb{N}^{l}$ such that $\vec{n} \cdot F^{\prime} \subset N(x, U)$. By Proposition 2.2 again, the set $F^{\prime}$ contains a geometric progression of length $k$, hence so does $N(x, U)$. Thus $x \in W_{k}$, moreover we have $X_{\varepsilon} \subset W_{k}$ as was to be shown.

Acknowledgements The authors would like to thank Professor X. Ye for helping discussions and remarks. The authors were supported by NNSF of China (11431012).

Open Access This article is licensed under a Creative Commons Attribution 4.0 International License, which permits use, sharing, adaptation, distribution and reproduction in any medium or format, as long as you give appropriate credit to the original author(s) and the source, provide a link to the Creative Commons licence, and indicate if changes were made. The images or other third party material in this article are included 
in the article's Creative Commons licence, unless indicated otherwise in a credit line to the material. If material is not included in the article's Creative Commons licence and your intended use is not permitted by statutory regulation or exceeds the permitted use, you will need to obtain permission directly from the copyright holder. To view a copy of this licence, visit http://creativecommons.org/licenses/by/4.0/.

\section{Appendix A. Basic Facts About Abstract Topological Dynamics}

In this appendix, we recall some basic definitions and results in abstract topological systems. For more details, see $[1,6]$.

\section{A.1. Topological Transformation Groups}

A topological dynamical system is a triple $\mathcal{X}=(X, \mathcal{T}, \Pi)$, where $X$ is a compact metrizable space, $\mathcal{T}$ is a $T_{2}$ topological group and $\Pi: T \times X \rightarrow X$ is a continuous map such that $\Pi(e, x)=x$ and $\Pi(s, \Pi(t, x))=\Pi(s t, x)$. We shall fix $\mathcal{T}$ and suppress the action symbol. In lots of literatures, $\mathcal{X}$ is also called a topological transformation group or a flow. Usually we omit $\Pi$ and demote a system by $(X, \mathcal{T})$.

Let $(X, \mathcal{T})$ be a system and $x \in X$, then $\mathcal{O}(x, \mathcal{T})$ denotes the orbit of $x$, which is also denoted by $\mathcal{T} x$. A subset $A \subset X$ is called invariant if $t a \in A$ for all $a \in A$ and $t \in \mathcal{T}$. When $Y \subset X$ is a closed and $\mathcal{T}$-invariant subset of the system $(X, \mathcal{T})$ we say that the system $(Y, \mathcal{T})$ is a subsystem of $(X, \mathcal{T})$. If $(X, \mathcal{T})$ and $(Y, \mathcal{T})$ are two dynamical systems their product system is the system $(X \times Y, \mathcal{T})$, where $t(x, y)=(t x, t y)$. A system $(X, \mathcal{T})$ is called minimal if $X$ contains no proper closed invariant subsets.

A homomorphism (or extension) of systems $\pi:(X, \mathcal{T}) \rightarrow(Y, \mathcal{T})$ is a continuous onto map of the phase spaces such that $\pi(t x)=t \pi(x)$ for all $t \in \mathcal{T}, x \in X$. In this case one says that $(Y, \mathcal{T})$ is a factor of $(X, \mathcal{T})$ and also that $(X, \mathcal{T})$ is an extension of $(Y, \mathcal{T})$. Define $R_{\pi}=\left\{\left(x_{1}, x_{2}\right): \pi\left(x_{1}\right)=\pi\left(x_{2}\right)\right\}$, then $Y=X / R_{\pi}$.

\section{A.2. Enveloping Semigroups}

Given a system $(X, \mathcal{T})$ its enveloping semigroup or Ellis semigroup $E(X, \mathcal{T})$ is defined as the closure of the set $\{t: t \in \mathcal{T}\}$ in $X^{X}$ (with its compact, usually non-metrizable, pointwise convergence topology). The maps $E \rightarrow E: p \mapsto p q$ and $p \mapsto t p$ are continuous for all $q \in E$ and $t \in \mathcal{T}$. Let $\pi: X \rightarrow Y$ be a factor map between systems $(X, \mathcal{T})$ and $(Y, \mathcal{T})$. Then there exists a unique continuous semigroup homomorphism $\pi^{*}: E(X, \mathcal{T}) \rightarrow E(Y, \mathcal{T})$ such that $\pi(p x)=\pi^{*}(p) \pi(x)$ for all $x \in X, p \in$ $E(X, \mathcal{T})$.

\section{A.3. Enveloping Semigroups Idempotents and Ideals}

For a semigroup the element $u$ with $u^{2}=u$ is called an idempotent. Ellis-Namakura Theorem says that for any enveloping semigroup $E$ the set $J(E)$ of idempotents of $E$ is not empty [6]. A non-empty subset $I \subset E$ is a left ideal (resp. right ideal) if $E I \subset I$ (resp. $I E \subset I$ ). A minimal left ideal is the left ideal that does not contain any proper 
left ideal of $E$. Obviously every left ideal is a semigroup and every left ideal contains some minimal left ideal.

We can introduce a quasi-order (a reflexive, transitive relation) $<_{L}$ on the set $J(E)$ by defining $v<_{L} u$ if and only if $v u=v$. If $v<_{L} u$ and $u<_{L} v$ we say that $u$ and $v$ are equivalent and write $u \sim_{L} v$. Similarly, we define $<_{R}$ and $\sim_{R}$. An idempotent $u \in J(E)$ is minimal if $v \in J(E)$ and $v<_{L} u$ implies $u<_{L} v$. The following results are well-known [4,8]: let $L$ be a left ideal of enveloping semigroup $E$ and $u \in J(E)$, then there is some idempotent $v$ in $L u$ such that $v<_{R} u$ and $v<_{L} u$; an idempotent is minimal if and only if it is contained in some minimal left ideal.

By using the method in [10], we can show the following result.

Theorem A.1 Let $(X, G)$ be a minimal system, where $G$ is an abelian group. Let $d \in \mathbb{N}$ and let $H$ be a subgroup of $G^{d}$. If $A$ is a $\Delta_{d}(G)$-minimal subset of $X^{d}$, put $N=\overline{\mathcal{O}(A, H)}=\overline{\bigcup\{S A: S \in H\}}$, then $\left(N, H^{\prime}\right)$ is a minimal system, where $H^{\prime}$ is the subgroup of $G^{d}$ generated by $\Delta_{d}(G)$ and $H$.

Proof Let $E=E\left(N, H^{\prime}\right)$ be the enveloping semigroup of $\left(N, H^{\prime}\right)$. Let $\pi_{i}: N \rightarrow X$ be the projection of $N$ on the $i$-th component, $i=1, \ldots, d$. We consider the action of the group $H^{\prime}$ on the $i$-th component. Thus $\pi_{i}$ is a factor map $\pi_{i}:\left(N, H^{\prime}\right) \rightarrow\left(X, H^{\prime}\right)$. Let $\pi_{i}^{*}: E\left(N, H^{\prime}\right) \rightarrow E\left(X, H^{\prime}\right)$ be the corresponding homomorphism of enveloping semigroups. Notice that for this action of $H^{\prime}$ on $X$ clearly $E\left(X, H^{\prime}\right)=E(X, G)$ as subsets of $X^{X}$.

Let now $u \in E\left(N, \Delta_{d}(G)\right)$ be any minimal idempotent in the enveloping semigroup of $\left(N, \Delta_{d}(G)\right)$. Choose $v$ a minimal idempotent in the closed left ideal $E\left(N, H^{\prime}\right) u$, then $v u=v$. For each $i=1,2, \ldots, d$, put $u_{i}=\pi_{i}^{*} u$ and $v_{i}=\pi_{i}^{*} v$. We want to show that also $u v=u$. Note that as an element of $E\left(N, H^{\prime}\right)$ is determined by its projections, it suffices to show that for each $i=1,2, \ldots, d, u_{i} v_{i}=u_{i}$.

Since for each $i=1,2, \ldots, d$ the map $\pi_{i}^{*}$ is a semigroup homomorphism, we have $v_{i} u_{i}=v_{i}$ as $v u=v$. In particular we deduce that $v_{i}$ is an element of the minimal left ideal of $E(X, G)$ which contains $u_{i}$. In turn this implies

$$
u_{i} v_{i}=u_{i} v_{i} u_{i}=u_{i}
$$

and it follows that indeed $u v=u$. Thus $u$ is an element of the minimal left ideal of $E\left(N, H^{\prime}\right)$ which contains $v$, and therefore $u$ is a minimal idempotent of $E\left(N, H^{\prime}\right)$.

Now let $\vec{x}$ be an arbitrary point in $A$ and let $u \in E\left(N, \Delta_{d}(G)\right)$ be a minimal idempotent with $u \vec{x}=\vec{x}$. By the above argument, $u$ is also a minimal idempotent of $E\left(N, H^{\prime}\right)$, whence $N=\overline{\mathcal{O}(A, H)}=\overline{\mathcal{O}\left(\vec{x}, H^{\prime}\right)}$ is $H^{\prime}$-minimal.

This completes the proof.

Now we are able to show Theorem 4.4 using the result above.

Proof of Theorem 4.4 Put $A=\Delta_{h}$ and $H=\left\langle T^{\mathbf{m}}\right\rangle$. It follows from Theorem A.1 immediately that $\left(X^{\Delta}, G\right)$ is minimal. 


\section{References}

1. Auslander, J.: Minimal Flows and Their Extensions. North-Holland Mathematics Studies, vol. 153. North-Holland, Amsterdam (1988)

2. Aliprantis, C.D., Borser, K.C.: Infinite Dimensional Analysis. A Hitchhiker's Guide, 3rd edn. Springer, Berlin (2006)

3. Brin, M., Stuck, G.: Introduction to Dynamical Systems. Cambridge University Press, Cambridge (2002)

4. Ellis, D., Ellis, R., Nerurkar, M.: The topological dynamics of semigroup actions. Trans. Am. Math. Soc. 353(4), 1279-1320 (2001)

5. Einsiedler, M., Ward, T.: Ergodic Theory with a View Towards Number Theory. Graduate Texts in Mathematics, vol. 259. Springer, London (2011)

6. Ellis, R.: Lectures on Topological Dynamics. W. A. Benjamin Inc, New York (1969)

7. Fort Jr., M.K.: Points of continuity of semi-continuous functions. Publ. Math. Debr. 2, 100-102 (1951)

8. Furstenberg, H., Katznelson, Y.: Idempotents in compact semigroups and Ramsey theory. Israel J. Math. 68, 257-270 (1989)

9. Glasscock, D., Koutsogiannis, A., Richter, F.K.: Multiplicative combinatorial properties of return time sets in minimal dynamical systems. Dis. Con. Dyn. Syst. 39(10), 5891-5921 (2019)

10. Glasner, E.: Structure theory as a tool in topological dynamics, descriptive set theory and dynamical systems (Marseille-Luminy, 1996), 173-209, London Mathematical Society Lecture Note Series, 277, Cambridge University Press, Cambridge (2000)

11. Szemerédi, E.: On sets of integers containing no $k$ elements in arithmetic progression. Acta Arith. 27, 299-345 (1975)

12. Ye, X.: D-function of a minimal set and an extension of Sharkovskii's theorem to minimal sets. Ergod. Theory Dyn. Syst. 12, 365-376 (1992) 